\title{
Note about canonical formalism for normalized gravity and vacuum energy sequestering model
}

\section{J. Klusoň}

Department of Theoretical Physics and Astrophysics, Faculty of Science, Masaryk University, Kotlárská 2, 611 3\%, Brno, Czech Republic

E-mail: klu@physics.muni.cz

ABStRact: This short note is devoted to the Hamiltonian analysis of the normalized general relativity and recently proposed model of vacuum energy sequestering. The common property of these models is the presence of the global variables. We discuss the meaning of these global variables in the context of the canonical formalism and argue that their presence lead to the non-local form of the Hamiltonian constraint.

KeYwords: Classical Theories of Gravity, Models of Quantum Gravity

ARXIV EPRINT: 1411.7501 


\section{Contents}

1 Introduction and summary 1

2 Hamiltonian analysis for systems with global variables 3

3 Normalized general relativity $\quad 6$

4 Hamiltonian analysis of vacuum energy sequestering model $\quad 8$

\section{Introduction and summary}

One of the most serious mystery of today's physics is the value of the cosmological constant [1]. There are many proposals how to explain the small values of given constant, for review of some of the most popular ones, see [2]. Another less known model that explains the origin of cosmological constant is unimodular gravity [6-17]. In the framework of unimodular gravity the cosmological constant $\Lambda$ is interpreted as an arbitrary constant of integration. However even if the cosmological constant is now determined by initial conditions we still do not know how to control its value.

Due to the fact that the value of the cosmological constant is very small one can wonder why it is not exactly equal to zero. In other words it would be interesting to find some symmetry principle that enforces the cosmological constant to be zero. Clearly the problem why the cosmological constant has so tiny value remains.

However the requirement that the cosmological constant is zero has remarkable consequence for the structure of the theory. In fact, this requirement can be enforced when we demand that the Lagrangian density is invariant under shift $\mathcal{L} \rightarrow \mathcal{L}+$ const. Clearly when the theory obeys this symmetry the cosmological constant is trivial. Then the requirement that the theory is invariant under constant shift of the Lagrangian leads to so called normalized Einstein-Hilbert action

$$
I=\frac{\int d^{4} x \sqrt{-\hat{g}}\left(\frac{1}{16 \pi G}{ }^{(4)} R+\mathcal{L}_{m}\right)}{\epsilon \int d^{4} x \sqrt{-\hat{g}}},
$$

where $\epsilon$ is the parameter of the mass dimension $M^{4}$ in order to make given action dimensionless. The above action was firstly introduced by Tseytlin [18] where he considered this theory as an effective low energy limit of some duality symmetric closed string theory. On the other hand given theory can be formulated without this assumption, see for example $[19,20]$. However it is important to stress that in the normalized general relativity framework the tree-level part of the cosmological constant is removed while the radiative corrections survive as was shown recently in [21]. This issue was solved recently in the model that was proposed in [21-23]. It is based on the mechanism that ensures that all 
the vacuum energy from a matter sector is sequestered from gravity. The idea it to make all scales in this matter sector functionals of the four volume element of the Universe. It turns out that the mechanism is minimal modification of general relativity when we add to the action auxiliary fields with an extra term that is not integrated over. Explicitly, this mechanism is described by the action

$$
S=\int d^{4} x \sqrt{-\hat{g}}\left[\frac{1}{16 \pi G}{ }^{(4)} R-\Lambda-\lambda^{4} \mathcal{L}\left(\lambda^{-2} \hat{g}^{\mu \nu}, \Phi\right)\right]+\sigma\left(\frac{\Lambda}{\lambda^{4} \mu^{4}}\right),
$$

when the matter with Lagrangian density $\mathcal{L}$ couples minimally to the rescaled metric $\tilde{g}_{\mu \nu}=\lambda^{2} \hat{g}_{\mu \nu}$. In this proposal $\Lambda$ and $\lambda$ are dynamical variables without any local dynamics. On the other hand the fact that the scales of matter sector are functionals of the four volume of the universe implies that in order to be non-zero the universe should be finite in spacetime so that it has to collapse in the future. The mechanism that leads to the collapse of the universe was proposed recently in [23]. Remarkably it turns out that given mechanism offers to solve some fundamental problems of recent cosmology, as for example famous "Why Now?" problem.

For that reason it is interesting to analyze given proposal from different point of views. It is important to stress that they are not conventional auxiliary fields where we can presume that they vary over the space-time but with no kinetic terms in the Lagrangian. Instead the variables used in the models above are global variables with no dependence of space-time coordinates. However this fact implies that even if given theory can be interpreted as a minimal modification of the general relativity the presence of the global variables has crucial impact on the Hamiltonian formulation. More precisely, the fact that these variables are global one implies that it is not possible to define their conjugate momenta. This is crucial difference from the case of auxiliary fields that appear in the Lagrangian density so that we can introduce their conjugate momenta as the primary constraints of the theory. We argue that in case of the global variables we should proceed in different way. First of all we introduce Hamiltonian for all dynamical fields that appear in the Lagrangian. Using this Hamiltonian and corresponding canonical variables we perform the variation of the action which leads to the equations of motion that can be expressed using conventional Poisson brackets. On the other hand the variation of the action with respect to the global variables leads to the set of relations between canonical variables. Note that by nature of these global variables these relations include the integration over the space and time. As the final step we include these relations to the equations of motion for the canonical variables. Say differently, if we express the equations of motion in terms of Poisson brackets we find that these global relations should be inserted to these Poisson brackets after their explicit evaluations. We demonstrate that this procedure is generally valid even for the system with constraints.

Following this general discussion we determine corresponding Hamiltonian structure for normalized general relativity and for the action (1.2). We firstly calculate the Poisson brackets between canonical variables and then insert the values of the global variables to them. Then it is clear that if we found that the Poisson bracket vanishes on the constraint surface it will vanish on the constraint surface even with the explicit values of the global 
variables included. In other words the constraint structure of all these theories is the same as in case of standard general relativity $[3,4]$. Of course, the problem is that the equations of motion for the canonical variables are non-local since they depend on the integrals of the canonical variables defined over the whole space-time. But when we presume that these global constraints can be fixed in some way we obtain well defined system with the clear constraint structure.

This paper is organized as follows. In the next section 2 we perform canonical analysis of general systems with global variables. Using this formalism we proceed to the Hamiltonian analysis of the normalized general relativity in section 3. Finally in section 4 we perform Hamiltonian analysis of the model proposed in [21-23].

\section{Hamiltonian analysis for systems with global variables}

Let us consider an action in the following form

$$
S=\int d t L(q, \dot{q}, \Lambda)+f(\Lambda)
$$

where $f$ is some function that depends on global variable $\Lambda$ and where $q$ generally means set of dynamical variables. The equations of motion for $q$ and $\Lambda$ that arise by variation of the action with respect to $q$ and $\Lambda$ take the form

$$
\frac{\delta L}{\delta q}-\frac{d}{d t}\left(\frac{\delta L}{\delta \dot{q}}\right)=0, \quad \int d t \frac{\delta L}{\delta \Lambda}+\frac{d f}{d \Lambda}=0 .
$$

Let us presume that the last equation can be solved for $\Lambda$ as a function of $q, \dot{q}$ so that $\Lambda=\Lambda\left(q^{\prime}, \dot{q}^{\prime}\right)$, where $q^{\prime} \equiv q\left(t^{\prime}\right), \dot{q}^{\prime}=\dot{g}\left(t^{\prime}\right)$ and where generally $\Lambda$ depends on $q^{\prime}, \dot{q}^{\prime}$ through the integral over $t^{\prime}$. Then inserting this value of $\Lambda$ into the first equation of (2.2) we derive the equation of motion for $q$ that is manifestly non-local.

Let us now try to formulate Hamiltonian formalism for given system. We define the canonical momentum $p$ and the Hamiltonian $H$ in the usual manner as $p=\frac{\delta L}{\delta \dot{q}}, H=$ $p \dot{q}-L$. Note that due to the fact that $\Lambda$ is global variable it does not make sense to define corresponding conjugate momentum. This can be also seen from the fact that this variable is not included in the Lagrangian of the theory. It is instructive to compare this situation with the possibility when $\Lambda$ depends on $t$ at least in principle. In this case we can introduce the conjugate momentum that, due to the fact that the Lagrangian does not depend on time derivative of $\Lambda$, is the primary constraint of the theory. The presence of such primary constraint would lead to the emergence of the secondary constraint which will be manifestly local.

Returning to our case we formulate the action using the canonical variables $p$ and $q$ so that it has the form

$$
S=\int d t(p \dot{q}-H(p, q, \Lambda))+f(\Lambda) .
$$

The variation of the action has the form

$$
\delta S=\int d t\left(\delta p \dot{q}-\dot{p} \delta q-\frac{\delta H}{\delta p} \delta p-\frac{\delta H}{\delta q} \delta q-\frac{\delta H}{\delta \Lambda} \delta \Lambda\right)+\frac{d f}{d \Lambda} \delta \Lambda=0
$$


so that we derive following equations of motion

$$
\begin{aligned}
\dot{q} & =\frac{\delta H(p, \Lambda)}{\delta p}, \\
\dot{p} & =-\frac{\delta H(p, \Lambda)}{\delta q}, \\
\int d t \frac{\delta H}{\delta \Lambda} & =\frac{d f}{d \Lambda} .
\end{aligned}
$$

Let us presume that the last equation can be solved for $\Lambda$, at least in principle, so that $\Lambda=\Lambda\left(p^{\prime}, q^{\prime}\right)$ Then we can insert this value to the first two equations and we derive

$$
\dot{q}(t)=\frac{\delta H\left(q, p, \Lambda\left(q^{\prime}, p^{\prime}\right)\right)}{\delta p}, \quad \dot{p}=-\frac{\delta H\left(q, p, \Lambda\left(q^{\prime}, p^{\prime}\right)\right)}{\delta q} .
$$

Note that this dependence of $\Lambda$ was introduced after the variation with respect to $q$ and $p$ was performed. Finally note the well known relation between variation of the Hamiltonian with respect to $q, p$ and the Poisson brackets so that we can rewrite these equations of motion into the form

$$
\begin{aligned}
& \dot{q}=\{q, H\}\left(\Lambda\left(q^{\prime}, p^{\prime}\right)\right), \\
& \dot{p}=\{p, H\}\left(\Lambda\left(q^{\prime}, p^{\prime}\right)\right),
\end{aligned}
$$

where again the explicit dependence of $\Lambda$ on $q^{\prime}, p^{\prime}$ is introduced after the calculation of the Poisson brackets. Clearly this procedure can be extended to any phase space functions $f(p, q)$.

On the other hand let us consider the more general case of systems with constraints. Let us presume that we have set of primary constraints $\phi_{n}(q, p, \Lambda) \approx 0$ that follow from the form of the Lagrangian. Note that generally $\phi_{n}$ depend on $\Lambda$. Then that extended Hamiltonian takes the form

$$
H_{E}=H+\lambda^{n} \phi_{n}(q, p, \Lambda)
$$

Now the variation principle takes the form

$$
\begin{aligned}
\delta S=\int d t & \delta p \dot{q}-\dot{p} \delta q-\frac{\delta H}{\delta p} \delta p-\frac{\delta H}{\delta q} \delta q-\frac{\delta H}{\delta \Lambda} \delta \Lambda- \\
& \left.-\lambda^{n} \frac{\delta \phi_{n}}{\delta q} \delta q-\lambda^{n} \frac{\delta \phi_{n}}{\delta p} \delta p-\lambda^{n} \frac{\delta \phi_{n}}{\delta \Lambda} \delta \Lambda\right)+\frac{d f}{d \Lambda} \delta \Lambda=0
\end{aligned}
$$

where we ignored the variation of the action with respect to $\lambda$ that implies the constraint $\phi_{n} \approx 0$. From this variation we derive the equation of motion

$$
\begin{aligned}
\dot{q} & =\frac{\delta H}{\delta p}+\lambda^{n} \frac{\delta \phi_{n}}{\delta p}, \\
\dot{p} & =-\frac{\delta H}{\delta q}-\lambda^{n} \frac{\delta \phi_{n}}{\delta q}, \\
\int d t\left[\frac{\delta H}{\delta \Lambda}+\lambda^{n} \frac{\delta \phi_{n}}{\delta \Lambda}\right] & =\frac{d f}{d \Lambda} .
\end{aligned}
$$


Let us for simplicity presume that $\phi_{n}$ does not depend on $\Lambda$. Then from the last equation we can find $\Lambda$, at least in principle. Further the requirement of the preservation of the constraints $\phi_{n}$ is equal to

$$
\frac{d \phi_{n}}{d t}=\left\{\phi_{n}, H\right\}(\Lambda)+\lambda^{m}\left\{\phi_{n}, \phi_{m}\right\}=0 .
$$

If $\left\{\phi_{n}, \phi_{m}\right\}$ is regular matrix then this equation can be solved for $\lambda^{m}$. On the other hand if $\left\{\phi_{n}, \phi_{m}\right\}$ is zero we determine another secondary constraints $\psi_{i}(p, q, \Lambda)$. We should include these constraints into the definition of the Hamiltonian so that we find the most general case where we have an extended Hamiltonian with collection of all constraints included where the constraints generally depend on $\Lambda$. Explicitly, let us denote $\Phi_{I}$ as the collection of all constraints, primary, secondary and so on so that the total Hamiltonian has the form ${ }^{1}$

$$
H_{T}=H+\lambda^{I} \Phi_{I}
$$

so that the extended action has the form

$$
S=\int d t\left(p \dot{q}-H_{T}\right)+f(\Lambda)=\int d t\left(p \dot{q}-H-\lambda^{I} \Phi_{I}\right)+f(\Lambda) .
$$

Then the requirement of the preservation of all constraints has the form

$$
\frac{d \Phi_{I}}{d t}=\left\{\Phi_{I}, H\right\}(\Lambda)+\lambda^{J}\left\{\Phi_{I}, \Phi_{J}\right\}=0 .
$$

Let us presume that these equations can be solved for $\lambda^{I}=\lambda^{I}(p, q, \Lambda)$. Then inserting these values of $\lambda^{I}$ into the "equation of motion" for $\Lambda$ that follows from (2.13)

$$
\int d t\left(\frac{\delta H}{\delta \Lambda}+\lambda^{I} \frac{\delta \Phi_{I}}{\delta \Lambda}\right)=\frac{d f}{d \Lambda}
$$

we find the equation for $\Lambda$ that can be solved for the canonical variables, at least in principle. Finally plugging this value of $\Lambda$ into $H$ and $\Phi^{I}$ we find non-local form of the Hamiltonian for given system. However it is important to stress that the constraint structure of given system does not depend on the explicit dependence of $\Lambda$ on the canonical variables as follows from the fact that we calculate the Poisson brackets with fixed $\Lambda$.

Let us also discuss the possibility that some of the Lagrange multipliers $\lambda$ were not fixed by equation (2.14) which would imply that corresponding constraints are the first class. Then we wound find that $\Lambda$ depends on non specified gauge parameters. On the other hand we can fix this gauge freedom by introducing corresponding set of gauge fixing functions that we denote as $\mathcal{G}_{\alpha}$ so that the extended action has the form

$$
S=\int d t\left(p \dot{q}-H-\Lambda^{I} \Phi_{I}-\omega^{\alpha} \mathcal{G}_{\alpha}\right)+f(\Lambda) .
$$

Then by definition of the gauge fixing functions we find that all Lagrange multipliers $\Lambda^{I}, \omega_{\alpha}$ are determined by the requirement of the preservation of the constraints $\phi_{I}, \mathcal{G}_{\alpha}$ during the time evolution of the system. Finally we determine $\Lambda$ from (2.15) with additional term $\omega^{\alpha} \frac{\delta \mathcal{G}_{\alpha}}{\delta \Lambda}$.

In the next two sections we apply this general analysis to the cases of two models introduced in the introduction section.

\footnotetext{
${ }^{1}$ For review of the Hamiltonian analysis of constrained systems, see [24, 25].
} 


\section{$3 \quad$ Normalized general relativity}

The normalized Einstein-Hilbert action has the form

$$
S=\frac{1}{\epsilon \int d^{4} x \sqrt{-\hat{g}}} \int d^{4} x \sqrt{-\hat{g}}\left(\frac{1}{16 \pi G}{ }^{(4)} R+\mathcal{L}_{\text {matter }}\right),
$$

where $\epsilon$ is constant with dimension $[\epsilon]=M^{4}$. To proceed to the Hamiltonian formalism we introduce two auxiliary global variables $A$ and $B$ and rewrite the action into the form

$$
S=\frac{1}{A} \int d^{4} x \sqrt{-\hat{g}}\left(\frac{1}{16 \pi G}^{(4)} R+\mathcal{L}_{\text {matter }}\right)+B\left(A-\epsilon \int d^{4} x \sqrt{-\hat{g}}\right) .
$$

The equations of motion that follow from this action have the form

$$
\begin{aligned}
A-\epsilon \int d^{4} x \sqrt{-\hat{g}} & =0, \\
-\frac{1}{A^{2}} \int d^{4} x \sqrt{-\hat{g}}\left(\frac{1}{16 \pi G}{ }^{(4)} R+\mathcal{L}_{\text {matter }}\right)+B & =0, \\
\frac{1}{A}\left[\frac{1}{16 \pi G}\left(R_{\mu \nu}-\frac{1}{2} \hat{g}_{\mu \nu} R\right)-T_{\mu \nu}\right]+\frac{1}{2} B \epsilon \hat{g}_{\mu \nu} & =0 .
\end{aligned}
$$

From the first equation we find $A=\epsilon \int d^{4} x \sqrt{-\hat{g}}$ so that the second equation gives

$$
B=\frac{1}{\left(\epsilon \int d^{4} x \sqrt{-\hat{g}}\right)^{2}} \int d^{4} x \sqrt{-\hat{g}}\left(\frac{1}{16 \pi G}^{(4)} R+\mathcal{L}_{\text {matter }}\right) .
$$

Inserting these two expressions to the equation of motion for $\hat{g}_{\mu \nu}$ we finally obtain

$$
\begin{aligned}
\frac{1}{16 \pi G}\left(R_{\mu \nu}-\frac{1}{2} \hat{g}_{\mu \nu} R\right)-T_{\mu \nu}+\frac{1}{2} \bar{S} \hat{g}_{\mu \nu} & =0, \\
\bar{S} & =\frac{\int d^{4} x \sqrt{-\hat{g}}\left(\frac{1}{16 \pi G}{ }^{(4)} R+\mathcal{L}_{\text {matter }}\right)}{\epsilon \int d^{4} x \sqrt{-\hat{g}}}
\end{aligned}
$$

that is the equation of motion found in [18]. To proceed to the canonical formulation we use the well know $3+1$ formalism that is the fundamental ingredient of the Hamiltonian formalism of any theory of gravity. ${ }^{2}$ We consider $3+1$ dimensional manifold $\mathcal{M}$ with the coordinates $x^{\mu}, \mu=0, \ldots, 3$ and where $x^{\mu}=(t, \mathbf{x}), \mathbf{x}=\left(x^{1}, x^{2}, x^{3}\right)$. We presume that this space-time is endowed with the metric $\hat{g}_{\mu \nu}\left(x^{\rho}\right)$ with signature $(-,+,+,+)$. Suppose that $\mathcal{M}$ can be foliated by a family of space-like surfaces $\Sigma_{t}$ defined by $t=x^{0}$. Let $g_{i j}, i, j=1,2,3$ denotes the metric on $\Sigma_{t}$ with inverse $g^{i j}$ so that $g_{i j} g^{j k}=\delta_{i}^{k}$. We further introduce the operator $\nabla_{i}$ that is covariant derivative defined with the metric $g_{i j}$. We also define the lapse function $N=1 / \sqrt{-\hat{g}^{00}}$ and the shift function $N^{i}=-\hat{g}^{0 i} / \hat{g}^{00}$. In terms of these variables we write the components of the metric $\hat{g}_{\mu \nu}$ as

$$
\begin{array}{lll}
\hat{g}_{00}=-N^{2}+N_{i} g^{i j} N_{j}, & \hat{g}_{0 i}=N_{i}, & \hat{g}_{i j}=g_{i j}, \\
\hat{g}^{00}=-\frac{1}{N^{2}}, & \hat{g}^{0 i}=\frac{N^{i}}{N^{2}}, & \hat{g}^{i j}=g^{i j}-\frac{N^{i} N^{j}}{N^{2}} .
\end{array}
$$

\footnotetext{
${ }^{2}$ For recent review, see [5].
} 
Using $3+1$ dimensional decomposition of the metric we find the primary constraints in the form

$$
\pi_{N} \approx 0, \pi_{i} \approx 0
$$

so that the bare Hamiltonian has the form

$$
\begin{aligned}
H & =\int d^{3} \mathbf{x}\left(N\left(\mathcal{H}_{T}+\epsilon B \sqrt{g}\right)+N^{i} \mathcal{H}_{i}\right) \\
\mathcal{H}_{T} & =\frac{16 \pi G A}{\sqrt{g}} \pi^{i j} \mathcal{G}_{i j k l} \pi^{k l}-\frac{\sqrt{g}}{16 \pi G A} R+\frac{A}{2 \sqrt{g}} p_{\Phi}^{2}+\frac{1}{2 A} \sqrt{g} g^{i j} \partial_{i} \Phi \partial_{j} \Phi+\frac{\sqrt{g}}{A} V(\Phi), \\
\mathcal{H}_{i} & =-2 g_{i k} \nabla_{j} \pi^{j k}+p_{\Phi} \partial_{i} \Phi
\end{aligned}
$$

where

$$
\mathcal{G}_{i j k l}=\frac{1}{2}\left(g_{i k} g_{j l}+g_{i l} g_{j k}\right)-\frac{1}{2} g_{i j} g_{k l},
$$

and where $R$ is three dimensional curvature. Further, $\pi^{i j}$ are momenta conjugate to $g_{i j}$ with non-zero Poisson bracket

$$
\left\{g_{i j}(\mathbf{x}), \pi^{k l}(\mathbf{y})\right\}=\frac{1}{2}\left(\delta_{i}^{k} \delta_{j}^{l}+\delta_{i}^{l} \delta_{j}^{k}\right) \delta(\mathbf{x}-\mathbf{y}) .
$$

Finally note that for simplicity we considered the matter Lagrangian density in the form

$$
\mathcal{L}_{\text {matter }}=-\frac{1}{2} \hat{g}^{\mu \nu} \partial_{\mu} \Phi \partial_{\nu} \Phi-V(\Phi)
$$

Now the preservation of the primary constraints imply an existence of the secondary constraints

$$
\mathcal{H}_{T}^{\prime} \equiv \mathcal{H}_{T}+\epsilon B \sqrt{g} \approx 0, \quad \mathcal{H}_{i} \approx 0
$$

Following the general discussion presented in previous section we should analyze the time evolution of all constraints. As usual it is useful to introduce the smeared form of the constraints $\mathcal{H}_{T}, \mathcal{H}_{i}$

$$
\mathbf{T}_{T}(X)=\int d^{3} \mathbf{x} X \mathcal{H}_{T}^{\prime}, \quad \mathbf{T}_{S}\left(X^{i}\right)=\int d^{3} \mathbf{x} X^{i} \mathcal{H}_{i},
$$

where $X, X^{i}$ are smooth functions on $\Sigma$ and where these constraints obey following Poisson bracket algebra

$$
\begin{aligned}
\left\{\mathbf{T}_{T}(X), \mathbf{T}_{T}(Y)\right\} & =\mathbf{T}_{S}\left(\left(X \partial_{i} Y-Y \partial_{i} X\right) g^{i j}\right), \\
\left\{\mathbf{T}_{S}(X), \mathbf{T}_{T}(Y)\right\} & =\mathbf{T}_{T}\left(X^{i} \partial_{i} Y\right) \\
\left\{\mathbf{T}_{S}\left(X^{i}\right), \mathbf{T}_{S}\left(Y^{j}\right)\right\} & =\mathbf{T}_{S}\left(X^{j} \partial_{j} Y^{i}-Y^{j} \partial_{j} X^{i}\right)
\end{aligned}
$$

From this algebra we see that these constraints are preserved under the time evolution of the system. Let us then consider the action

$$
S=\int d t d^{3} \mathbf{x}\left(\pi^{i j} \partial_{t} g_{i j}-N\left(\mathcal{H}_{T}+\epsilon B \sqrt{g}\right)-N^{i} \mathcal{H}_{i}\right)+B A
$$


so that the equation of motion with respect to $A$ and $B$ have the form

$$
\begin{aligned}
A & =\epsilon \int d t d^{3} \mathbf{x} N \sqrt{g}, \\
B & =\int d t d^{3} \mathbf{x} N \frac{\delta \mathcal{H}_{T}}{\delta A} \\
& =\int d t d^{3} \mathbf{x} N\left(\frac{16 \pi G}{\sqrt{g}} \pi^{i j} \mathcal{G}_{i j k l} \pi^{k l}+\frac{\sqrt{g}}{16 \pi G A^{2}} R+\frac{1}{2 \sqrt{g}} p_{\Phi}^{2}-\frac{1}{2 A^{2}} \sqrt{g} g^{i j} \partial_{i} \Phi \partial_{j} \Phi-\frac{\sqrt{g}}{A^{2}} V(\Phi)\right) .
\end{aligned}
$$

Finally inserting these values to the Hamiltonian constraint $\mathcal{H}_{T}^{\prime}$ we obtain the Hamiltonian form of the normalized general relativity where the Hamiltonian constraint is manifestly non-local but which, according to the discussion given above, has the same constraint structure as general relativity with the collection of the first class constraints $\pi_{N} \approx 0, \pi_{i} \approx$ $0, \mathcal{H}_{T}^{\prime} \approx 0, \mathcal{H}_{i} \approx 0$. These constraints could be eventually fixed by some gauge fixing procedure with corresponding consequence for the values of the global variables $A$ and $B$.

\section{Hamiltonian analysis of vacuum energy sequestering model}

In this section we would like to perform Hamiltonian analysis of the model proposed in [21]. The action has the form

$$
S=\int d^{4} x \sqrt{-\hat{g}}\left[\frac{M_{P l}^{2}}{2}{ }^{(4)} R-\Lambda-\lambda^{4} \mathcal{L}_{\text {matter }}\left(\lambda^{-2} \hat{g}^{\mu \nu}, \Phi\right)\right]+\sigma\left(\frac{\Lambda}{\lambda^{4} \mu^{4}}\right),
$$

where matter couples minimally to the rescaled metric $\tilde{g}_{\mu \nu}=\lambda^{2} \hat{g}_{\mu \nu}$. For simplicity we again consider the matter action given in (3.11). Finally $\Lambda$ and $\lambda$ that appear in (4.1) are global variables.

In the similar way as in previous section we find the Hamiltonian in the form

$$
H=\int d^{3} \mathbf{x}\left(N\left(\mathcal{H}_{T}+\sqrt{g} \Lambda\right)+N^{i} \mathcal{H}_{i}\right)
$$

with the primary constraints of the theory

$$
\pi_{N} \approx 0, \pi_{i} \approx 0
$$

and where

$$
\begin{aligned}
\mathcal{H}_{T} & =\frac{2}{M_{p l}^{2} \sqrt{g}} \pi^{i j} \mathcal{G}_{i j k l} \pi^{k l}-\frac{M_{p l}^{2}}{2} \sqrt{g} R+\frac{1}{2 \lambda^{2} \sqrt{g}} p_{\Phi}^{2}+\frac{\lambda^{2}}{2} \sqrt{g} g^{i j} \partial_{i} \Phi \partial_{j} \Phi+\lambda^{4} V(\Phi), \\
\mathcal{H}_{i} & =-2 g_{i k} \nabla_{j} \pi^{j k}+p_{\Phi} \partial_{i} \Phi
\end{aligned}
$$

Now preservation of the primary constraints imply the secondary constraints

$$
\begin{aligned}
\partial_{t} \pi_{N} & =\left\{\pi_{N}, H\right\}=-\mathcal{H}_{T}-\sqrt{g} \Lambda-\equiv-\mathcal{H}_{T}^{\prime} \approx 0, \\
\partial_{t} \pi_{i} & =\left\{\pi_{i}, H\right\}=-\mathcal{H}_{i} \approx 0 .
\end{aligned}
$$


Note that the smeared form of these constraints have the same Poisson brackets as in (3.14) so that given theory has the same constraint structure. Now the corresponding action has the form

$$
S=\int d t d^{3} \mathbf{x}\left(\pi^{i j} \dot{g}_{i j}-N\left(\mathcal{H}_{T}+\sqrt{g} \Lambda\right)-N^{i} \mathcal{H}_{i}\right)+\sigma\left(\frac{\Lambda}{\lambda^{4} \mu^{4}}\right),
$$

where $\Lambda, \lambda$ are determined from the equations

$$
\begin{aligned}
\frac{1}{\mu^{4} \lambda^{4}} \sigma^{\prime} & =\int d t d^{3} \mathbf{x} N \sqrt{g} \\
4 \frac{\Lambda}{\lambda^{5} \mu^{4}} \sigma^{\prime} & =-\int d t d^{3} \mathbf{x}\left(-\frac{1}{\lambda^{3} \sqrt{g}} p_{\Phi}^{2}+\lambda \sqrt{g} g^{i j} \partial_{i} \Phi \partial_{j} \Phi+4 \lambda^{3} V(\Phi)\right),
\end{aligned}
$$

where $\sigma^{\prime}(x) \equiv \frac{d \sigma}{d x}$. As was argued in [21,22] the form of the function $\sigma$ should be determined on phenomenological grounds but in principle we can presume that equations given above can be solved for $\lambda, \Lambda$. For simplicity let us consider the simplest case $\sigma(x)=x$. Then from the first equation we obtain

$$
\lambda^{4}=\frac{1}{\mu^{4} \int d t d^{3} \mathbf{x} N \sqrt{g}}
$$

and from the second one we obtain

$$
\Lambda=\frac{1}{4} \int d t d^{3} \mathbf{x}\left(\frac{\lambda^{2}}{\sqrt{g}} p_{\Phi}^{2}-\lambda^{6} \sqrt{g} g^{i j} \partial_{i} \Phi \partial_{j} \Phi-4 \lambda^{4} V(\Phi)\right) .
$$

Inserting these values to the Hamiltonian we find the final form of the Hamiltonian formulation of this model that has similar canonical structure as the normalized general relativity.

\section{Acknowledgments}

This work was supported by the Grant Agency of the Czech Republic under the grant P201/12/G028.

Open Access. This article is distributed under the terms of the Creative Commons Attribution License (CC-BY 4.0), which permits any use, distribution and reproduction in any medium, provided the original author(s) and source are credited.

\section{References}

[1] S. Weinberg, The Cosmological Constant Problem, Rev. Mod. Phys. 61 (1989) 1 [inSPIRE].

[2] E.J. Copeland, M. Sami and S. Tsujikawa, Dynamics of dark energy, Int. J. Mod. Phys. D 15 (2006) 1753 [hep-th/0603057] [INSPIRE].

[3] C.J. Isham and K.V. Kuchar, Representations of Space-time Diffeomorphisms. 2. Canonical Geometrodynamics, Annals Phys. 164 (1985) 316 [INSPIRE].

[4] C.J. Isham and K.V. Kuchar, Representations of Space-time Diffeomorphisms. 1. Canonical Parametrized Field Theories, Annals Phys. 164 (1985) 288 [INSPIRE].

[5] E. Gourgoulhon, $3+1$ formalism and bases of numerical relativity, gr-qc/0703035 [INSPIRE]. 
[6] G.F.R. Ellis, H. van Elst, J. Murugan and J.-P. Uzan, On the Trace-Free Einstein Equations as a Viable Alternative to General Relativity, Class. Quant. Grav. 28 (2011) 225007 [arXiv: 1008.1196] [INSPIRE].

[7] G.F.R. Ellis, The Trace-Free Einstein Equations and inflation, Gen. Rel. Grav. 46 (2014) 1619 [arXiv: 1306.3021] [INSPIRE].

[8] A. Padilla and I.D. Saltas, A note on classical and quantum unimodular gravity, arXiv: 1409.3573 [INSPIRE].

[9] C. Gao, R.H. Brandenberger, Y. Cai and P. Chen, Cosmological Perturbations in Unimodular Gravity, arXiv:1405.1644 [INSPIRE].

[10] P. Jain, A. Jaiswal, P. Karmakar, G. Kashyap and N.K. Singh, Cosmological implications of unimodular gravity, JCAP 11 (2012) 003 [arXiv:1109.0169] [INSPIRE].

[11] L. Smolin, Unimodular loop quantum gravity and the problems of time, Phys. Rev. D 84 (2011) 044047 [arXiv: 1008.1759] [INSPIRE].

[12] L. Smolin, The Quantization of unimodular gravity and the cosmological constant problems, Phys. Rev. D 80 (2009) 084003 [arXiv:0904.4841] [InSPIRE].

[13] M. Shaposhnikov and D. Zenhausern, Scale invariance, unimodular gravity and dark energy, Phys. Lett. B 671 (2009) 187 [arXiv:0809.3395] [INSPIRE].

[14] E. Alvarez, Can one tell Einstein's unimodular theory from Einstein's general relativity?, JHEP 03 (2005) 002 [hep-th/0501146] [INSPIRE].

[15] D.R. Finkelstein, A.A. Galiautdinov and J.E. Baugh, Unimodular relativity and cosmological constant, J. Math. Phys. 42 (2001) 340 [gr-qc/0009099] [INSPIRE].

[16] C. Barceló, R. Carballo-Rubio and L.J. Garay, Unimodular gravity and general relativity from graviton self-interactions, Phys. Rev. D 89 (2014) 124019 [arXiv:1401.2941] [INSPIRE].

[17] C. Barceló, R. Carballo-Rubio and L.J. Garay, Absence of cosmological constant problem in special relativistic field theory of gravity, arXiv:1406.7713 [INSPIRE].

[18] A.A. Tseytlin, Duality symmetric string theory and the cosmological constant problem, Phys. Rev. Lett. 66 (1991) 545 [INSPIRE].

[19] A. Davidson and S. Rubin, Normalized general relativity: Nonclosed universe and a zero cosmological constant, Phys. Rev. D 89 (2014) 024036 [arXiv:1401.8113] [INSPIRE].

[20] A. Davidson and S. Rubin, Zero Cosmological Constant from Normalized General Relativity, Class. Quant. Grav. 26 (2009) 235019 [arXiv:0905.0661] [INSPIRE].

[21] N. Kaloper and A. Padilla, Sequestering the Standard Model Vacuum Energy, Phys. Rev. Lett. 112 (2014) 091304 [arXiv: 1309.6562] [INSPIRE].

[22] N. Kaloper and A. Padilla, Vacuum Energy Sequestering: The Framework and Its Cosmological Consequences, Phys. Rev. D 90 (2014) 084023 [arXiv:1406.0711] [InSPIRE].

[23] N. Kaloper and A. Padilla, 'The End', arXiv:1409.7073 [inSPIRE].

[24] J. Govaerts, The Quantum geometer's universe: Particles, interactions and topology, hep-th/0207276 [INSPIRE].

[25] M. Henneaux and C. Teitelboim, Quantization of gauge systems, Princeton, Univ. Pr., 1992, pg. 520, U.S.A. 